\title{
ADRC Method for Noncascaded Integral System Based on the Total Derivative of Composite Functions of Several Variables
}

\author{
Zhijian Huang, ${ }^{1,2}$ Xuemei Xiong, ${ }^{1}$ Hailong Wang, ${ }^{1}$ Yuli Chen, ${ }^{1}$ and Yihua Liu' \\ ${ }^{1}$ Merchant Marine College, Shanghai Maritime University, Shanghai 201306, China \\ ${ }^{2}$ Institute of Power Plant and Automation, Shanghai Jiao Tong University, Shanghai 200030, China \\ Correspondence should be addressed to Zhijian Huang; zjhuang@shmtu.edu.cn
}

Received 31 May 2016; Revised 13 October 2016; Accepted 30 October 2016

Academic Editor: Rafael Morales

Copyright (c) 2016 Zhijian Huang et al. This is an open access article distributed under the Creative Commons Attribution License, which permits unrestricted use, distribution, and reproduction in any medium, provided the original work is properly cited.

\begin{abstract}
The standard ADRC controller usually selects the canonical plant in the form of cascaded integrators. However, the condition variables of practical system do not necessarily have the cascaded integral relationship. Therefore, this paper proposes a method of total derivative of composite functions of several variables and a structure, which can convert the state space system of noncascaded integral form into the cascaded integral form. In this way, the converted system can be directly controlled with ADRC. Meanwhile, the control of Chen chaotic system is discussed in detail to show the conversion and the controller design. The control performances under different levels of complication and different strengths of disturbance are comparably researched. The converted system achieves significantly better control effects under ADRC than that of the PID. This converting method solves the control problem of some noncascaded integral systems in both theory and application and greatly expands the application scope of the standard ADRC method.
\end{abstract}

\section{Introduction}

The Active Disturbance Rejection Control (ADRC) method has begun to attract more and more attention and have been widely used in many areas in recent years [1-23], because of its novel concept, simple implementation, and superior performance in feedback control. The ADRC theory was introduced by Han $[24,25]$ and significantly improved both the control quality and the control accuracy where PID can be used [25].

However, the ADRC selects the canonical plant as the cascaded integral form. The existing standard ADRC is only available to cascaded integral systems that satisfy the so-called matching conditions, such as the motion control system [10]. ADRC uses extended state observer (ESO) to estimate the object states and its disturbances, which has cascaded integral character. This character makes the ADRC more suitable to exert its unique control effect for cascaded integral systems. However, the canonical form of the cascaded integral system was usually misconstrued as the only form in which ADRC can be used. In fact, the practical system is not always the cascaded integral form. Thus, it is necessary to study how to design ADRC for other forms of system which does not satisfy the matched conditions or replace the PID for the control of state space systems.

At present, the research situation in this area is as follows: (1) some applications are used as converting method. A converting method is differential geometry $[26,27]$. This method is based on the model of the controlled object and combines the nonlinear state conversion and linearization. Huang and Xue used algebraic substitution to obtain one of the partial derivatives, but the second-order state is not converted [27], so it is not a strict conversion method. For the multiorder state space system, Huang and Xue also used the same method, which also has the same situation [27]. RamírezNeria et al. used the decoupling property of object model and naturally decomposed it into a cascaded connection of two independent blocks [28], which simplifies the observer design in ADRC. (2) Some applications are only limited to linear system where the controlled object can be converted into cascaded integral system, that is, the controlled object of differential equation, rational proper fraction, or state space 


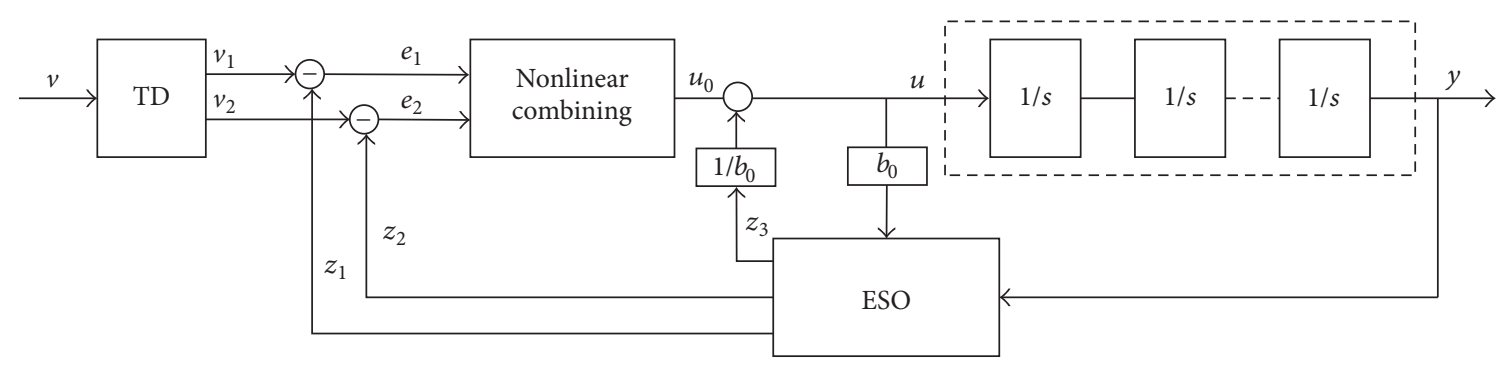

FIGURE 1: The structure diagram of cascaded integral ADRC system expressed in the form of transfer function.

form, such as Refs $[12,13,29,30]$. So, the application scope is limited. (3) Some applications are only limited to controlled object system of cascaded expression form; for example, the nonlinear ADRC has been successfully applied to the fast tool servosystem $[14,31]$. This is a cascaded integral system of two stages. So, the application scope is even more limited. (4) A lot of applications are reduced to a pure cascaded integral plant with closed-loop feedback. This is just the original function of ADRC. It does not treat object form before controller design; for example, Wu et al. used ESO and linear ADRC feedback to control a fast tool servosystem [10]. Feng and Guo adopted the ADRC control approach to stabilize a system described by the partial differential equation with corrupted output feedback [32]. Yang, Sun, Zhang, and Huang et al. used similar methods and had the similar situations $[7,8,15$, 16]. (5) In [33], the nonminimum phase system is actually a noncascaded integral system. The reference presents a combined feedforward and model-assisted ADRC strategy. It has only one tuning parameter but needs a nominal model.

However, the actual controlled systems have various forms. The system needs to be converted to a typical paradigm of ADRC to adapt to its control and better play its control effect when not necessarily conforming to the typical cascaded integral case. Thus, there may be a lot of styles to be converted, and the conversion method may not be the same. To the best of our knowledge, the state space system is one of the most widely expressed forms in practical systems. If the system, expressed as state space form or as state space form, is converted into cascaded integral system suitable for ADRC application, it will be highly representative.

In this paper, the method of total derivative of composite functions of several variables and the structure is used to convert system, expressed as state space form or as state space form, into cascaded integral system suitable for ADRC application. Since this conversion will not be influenced by the accuracy of the object model estimation, for state space object system of noncascaded integral form with unknown object model, it can still realize the conversion and its ADRC control. The error of the object model can be seen as an internal or external disturbance for ADRC controller and then be estimated and compensated by the ESO. This is also a great advantage of ADRC controller. The conversion for state space controlled object with noncascaded integral form has a certain degree of representation and it can solve the conversion problem of many systems. Also, the feedback control effect of ADRC for many noncascaded integral systems can be greatly improved. Thus, the application scope of ADRC method is also greatly expanded.

\section{ADRC Method for Cascaded Integral System}

The so-called cascaded integral system is a closed state feedback system, described by state equation

$$
\begin{gathered}
\dot{x}_{1}=x_{2}, \\
\vdots \\
\dot{x}_{n-1}=x_{n}, \\
\dot{x}_{n}=b(t) u(t), \\
y=x_{1}(t) .
\end{gathered}
$$

In it, $x_{i}, i=1,2, \ldots, n$ are the state variables of controlled system; $u$ is the control input; $y$ is the system output. $b(t) \in R^{m}$ is nonlinear and possibly unknown, and $b(t)$ is nonsingular. If the ADRC feedback control system of above states is expressed with structure graph, the controlled object is series form of $n$ integral part of $1 / s$, as shown within the gridlines in Figure 1. This kind of cascaded integral system is the typical form suitable for ADRC method.

The ADRC method is also shown in Figure 1, which perfectly overcomes many shortcomings of a closed-loop feedback control, and realizes nonlinear control. The core idea is that both the internal dynamics and external disturbances of controlled system can be estimated and compensated in real time. It adopts the following four steps: (1) arranging transition process for control target with tracking differentiation (TD); (2) estimating the state and total disturbance of controlled object with ESO; (3) nonlinear feedback law of state errors; and (4) disturbance compensation process. The detail of ADRC method is in [24, 25, 27].

\section{The Structure Method of the Total Derivative of Several Variables Composite Functions}

By the structure method of total derivative of composite functions of several variables, the state space object system of noncascaded integral form can be converted into cascaded integral system suitable for ADRC controller. Thus, it extends 
the application scope of standard ADRC method, no longer limited to the cascaded integral system.

Lemma 1. For the case where composite function has several intermediate variables, set $z=f[u, v, \omega], u=\varphi(t), v=$ $\psi(t)$, and $\omega=\omega(t)$ and then get the composite function $z=$ $f[\varphi(t), \psi(t), \omega(t)]$. If the functions $u=\varphi(t), v=\psi(t)$, and $\omega=$ $\omega(t)$ are all differentiable at $t$ point, and function $z=f[u, v, \omega]$ has a continuous partial derivative at the corresponding point $(u, v, \omega)$, then, the composite function $z=f[\varphi(t), \psi(t), \omega(t)]$ is also differentiable at $t$ point and has

$$
\frac{d z}{d t}=\frac{\partial z}{\partial u} \frac{d u}{d t}+\frac{\partial z}{\partial v} \frac{d v}{d t}+\frac{\partial z}{\partial \omega} \frac{d \omega}{d t}
$$

Then, the derivative $d z / d t$ in (2) is called the total derivative of composite functions of several variables [34].

For a given nonlinear time-varying dynamic system with noncascaded integral form, it has three state variables $x_{1}, x_{2}, x_{3}$, the output variable $y=x_{1}$, and the input variable $u$, shown as follows:

$$
\begin{aligned}
& \dot{x}_{1}=f_{1}\left(x_{1}, x_{2}, x_{3}\right), \\
& \dot{x}_{2}=f_{2}\left(x_{1}, x_{2}, x_{3}\right)+u, \\
& \dot{x}_{3}=f_{3}\left(x_{1}, x_{2}, x_{3}\right), \\
& y=x_{1} .
\end{aligned}
$$

Assume that $f_{1}\left(x_{1}, x_{2}, x_{3}\right), f_{2}\left(x_{1}, x_{2}, x_{3}\right)$, and $f_{3}\left(x_{1}, x_{2}, x_{3}\right)$ in (3) are all continuous and differentiable, and the system state variables can be observed. In order to construct a cascaded integral system, set $z=f_{1}\left(x_{1}, x_{2}, x_{3}\right)$, then define $d z / d t=$ $g\left(x_{1}, x_{2}, x_{3}\right)$, and thus construct the total derivative equation of composite functions of several variables as follows:

$$
\begin{aligned}
\frac{d z}{d t}= & g\left(x_{1}, x_{2}, x_{3}\right)=\frac{\partial z}{\partial x_{1}} \frac{d x_{1}}{d t}+\frac{\partial z}{\partial x_{2}} \frac{d x_{2}}{d t}+\frac{\partial z}{\partial x_{3}} \frac{d x_{3}}{d t} \\
= & \frac{\partial z}{\partial x_{1}} f_{1}\left(x_{1}, x_{2}, x_{3}\right)+\frac{\partial z}{\partial x_{2}}\left(f_{2}\left(x_{1}, x_{2}, x_{3}\right)+u\right) \\
& +\frac{\partial z}{\partial x_{3}} f_{3}\left(x_{1}, x_{2}, x_{3}\right) .
\end{aligned}
$$

Meanwhile, carry out a coordinate transformation for (4), and set up a new nonlinear time-varying dynamic system:

$$
\begin{aligned}
& y_{1}=x_{1}, \\
& y_{2}=z=f_{1}\left(x_{1}, x_{2}, x_{3}\right), \\
& y_{3}=g\left(x_{1}, x_{2}, x_{3}\right) .
\end{aligned}
$$

Thus, the nonlinear time-varying dynamic system of cascaded integral form can be constructed according to the above equations, shown as follows:

$$
\begin{aligned}
& \dot{y}_{1}=y_{2}, \\
& \dot{y}_{2}=y_{3}, \\
& \dot{y}_{3}=\frac{\partial g}{\partial x_{1}} \dot{x}_{1}+\frac{\partial g}{\partial x_{2}} \dot{x}_{2}+\frac{\partial g}{\partial x_{3}} \dot{x}_{3}, \\
& y=y_{1} .
\end{aligned}
$$

After arrangement and simplification, then get

$$
\begin{aligned}
\dot{y}_{1}= & y_{2}, \\
\dot{y}_{2}= & y_{3}, \\
\dot{y}_{3}= & \frac{\partial g}{\partial x_{1}} f_{1}\left(x_{1}, x_{2}, x_{3}\right)+\frac{\partial g}{\partial x_{2}}\left(f_{2}\left(x_{1}, x_{2}, x_{3}\right)+u\right) \\
& +\frac{\partial g}{\partial x_{3}} f_{3}\left(x_{1}, x_{2}, x_{3}\right), \\
y= & y_{1} .
\end{aligned}
$$

\section{Given Controlled Object}

For the following system of noncascaded integral form, namely, the Chen chaotic system,

$$
\begin{aligned}
& \dot{x}_{1}=p\left(x_{2}-x_{1}\right), \\
& \dot{x}_{2}=(m-p) x_{1}-x_{1} x_{3}+m x_{2}+u+\omega, \\
& \dot{x}_{3}=x_{1} x_{2}-n x_{3}, \\
& y=x_{1} .
\end{aligned}
$$

In it, $p, m$, and $n$ are system parameters; $u$ is the control input; $\omega=\sin (t)$ is the added internal or external disturbance signal or $\omega=0$ means no internal and external disturbance. When the parameters and state variables are set as $p=35$, $m=28, n=3, x_{1}(0)=9, x_{2}(0)=-1$, and $x_{3}(0)=0$, the Chen system shows chaotic condition. The time response and attractor phase space of the Chen chaotic system are shown in Figure 2.

The Chen chaotic system can be controlled by the following self-feedback method, which makes the state variables converge to equilibrium state on zero points.

Lemma 2. A controlled object of Chen chaotic system is designed as

$$
\begin{aligned}
& \dot{x}_{1}=p\left(x_{2}-x_{1}\right), \\
& \dot{x}_{2}=(m-p) x_{1}-x_{1} x_{3}+m x_{2}-k x_{2}, \\
& \dot{x}_{3}=x_{1} x_{2}-n x_{3}, \\
& y=x_{1} .
\end{aligned}
$$

When $k>(2 m-p)$, the controlled Chen chaotic system is locally asymptotically stable at $S_{0}(0,0,0)$ [35]. 


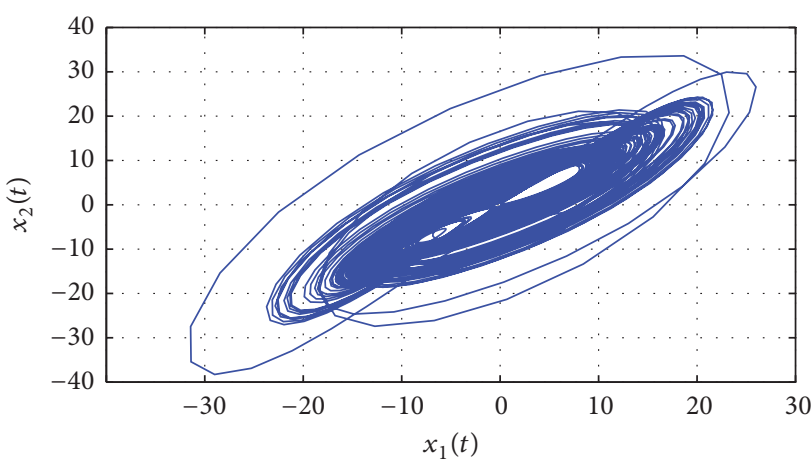

(a)

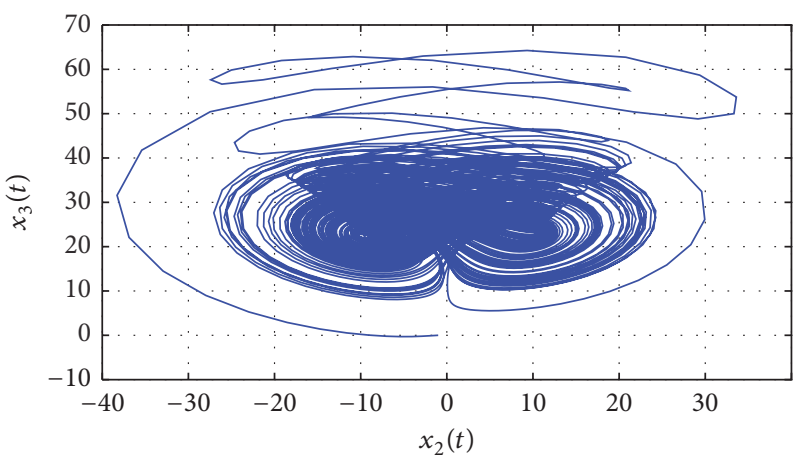

(c)

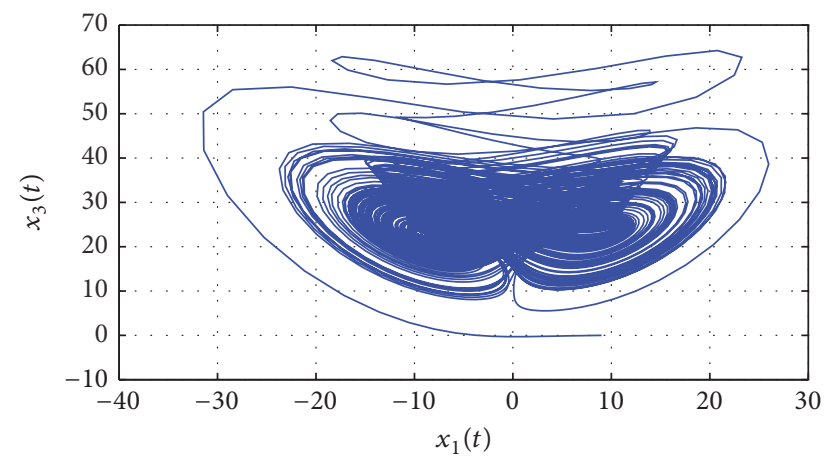

(b)

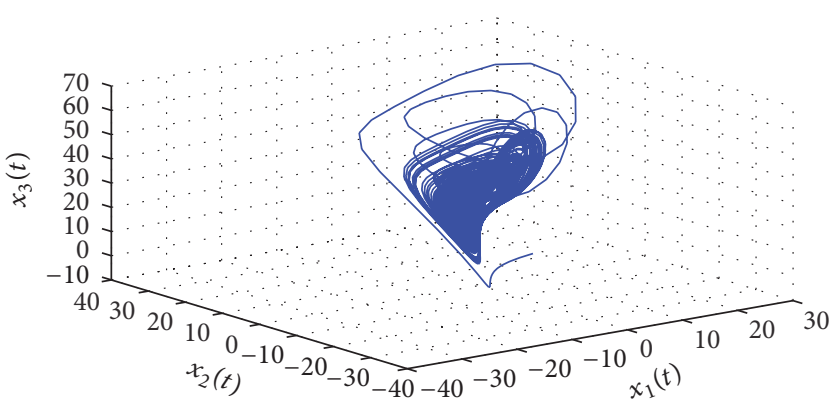

(d)

Figure 2: The time response and attractor phase space of the Chen chaotic system from initial states $x_{1}(0)=9, x_{2}(0)=-1$, and $x_{3}(0)=0$. In it, (a) the projection graph on $x_{1} x_{2}$ plane; (b) the projection graph on $x_{1} x_{3}$ plane; (c) the projection graph on $x_{2} x_{3}$ plane; and (d) the projection graph on $x_{1} x_{2} x_{3}$ plane.

So, one of the methods to control Chen chaotic system is to use PID or ADRC controller. The controller generates the appropriate $k$ value, so the Chen chaotic system can quickly and stably converge to the zero equilibrium point.

\section{PID Control and Experiment for the Original System}

5.1. The Design of PID Controller. For the controlled object in (8), its error feedback controller of PID form can be designed as

$$
\begin{aligned}
& e=v_{0}-x, \\
& u=K\left(\frac{1}{T_{i}} \int_{0}^{t} e(t) d t+e+T_{d} \dot{e}\right) .
\end{aligned}
$$

In it, the set value is taken as the convergence signal on zero equilibrium point of $v_{0}(t)=0$. The PID parameters of the best control effect are found, by the trial and error method, as $K=1, T_{i}=1$, and $T_{d}=1$.

\subsection{The PID Control Effect for the Original System}

(1) When the internal/external disturbances are all $\omega=0$ and the PID control parameters are $K=1, T_{i}=$ $1, T_{d}=1$, the best control effect that can be achieved for Chen chaotic system from initial states $x_{1}(0)=9$, $x_{2}(0)=-1$, and $x_{3}(0)=0$ is shown in Figure 3.

(2) Under the same PID controller and its parameter settings, when there is an internal disturbance signal of $\omega=\sin (t)$, the best control effect that can be achieved for Chen chaotic system from initial states $x_{1}(0)=9, x_{2}(0)=-1$, and $x_{3}(0)=0$ is shown in Figure 4 .

(3) Under the same PID controller and its parameter settings, when there is an external disturbance signal of $\omega=\sin (t)$, the best control effect that can be achieved for Chen chaotic system from initial states $x_{1}(0)=9, x_{2}(0)=-1$, and $x_{3}(0)=0$ is shown in Figure 5.

\section{ADRC Control and Experiment for the Converted System}

6.1. The Design of ADRC Controller. Now, construct (8) into a cascaded integral system adopting the total derivative of several variables composite functions method in Section 3, that is, according to (3) to (7). After arrangement, the ADRC 


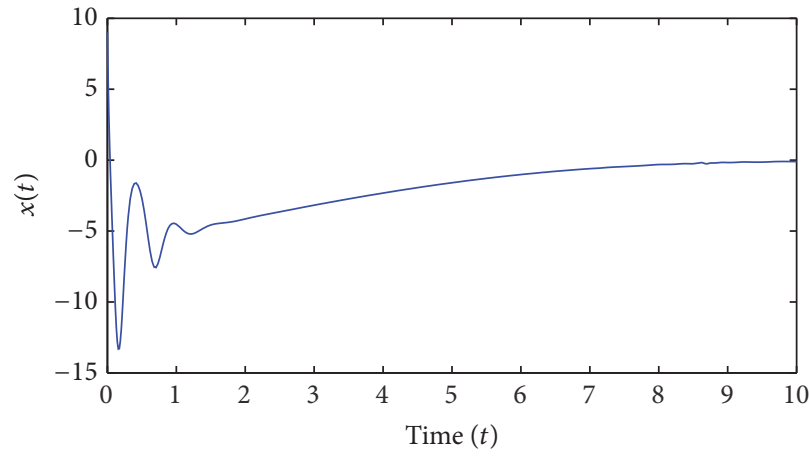

(a)

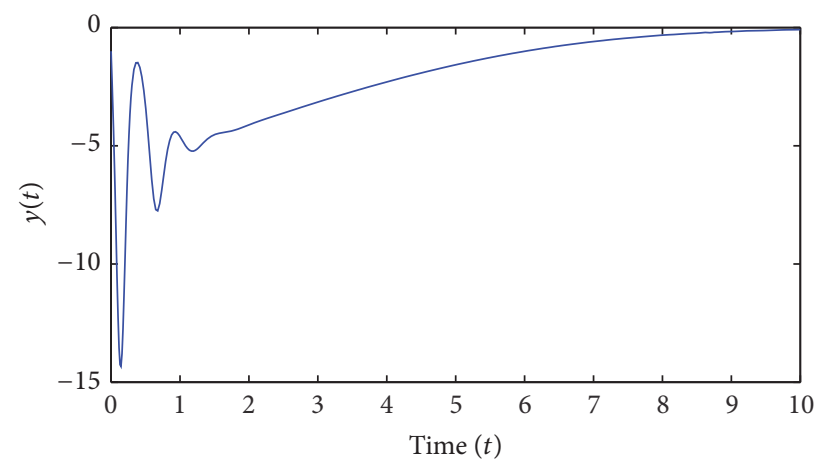

(b)

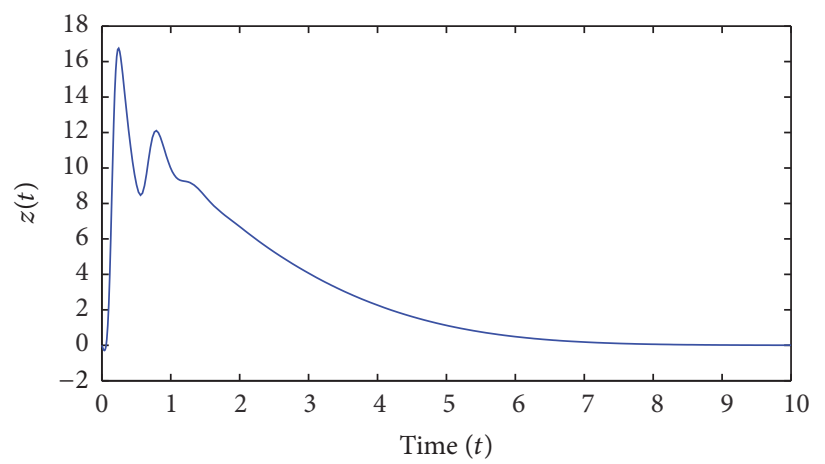

(c)

FIGURE 3: The PID control effect on Chen chaotic system without disturbing from initial states $x_{1}(0)=9, x_{2}(0)=-1$, and $x_{3}(0)=0$. In it, (a) the control effect of PID on $x_{1}$ variable of Chen chaotic system; (b) the control effect of PID on $x_{2}$ variable of Chen chaotic system; (c) and the control effect of PID on $x_{3}$ variable of Chen chaotic system.

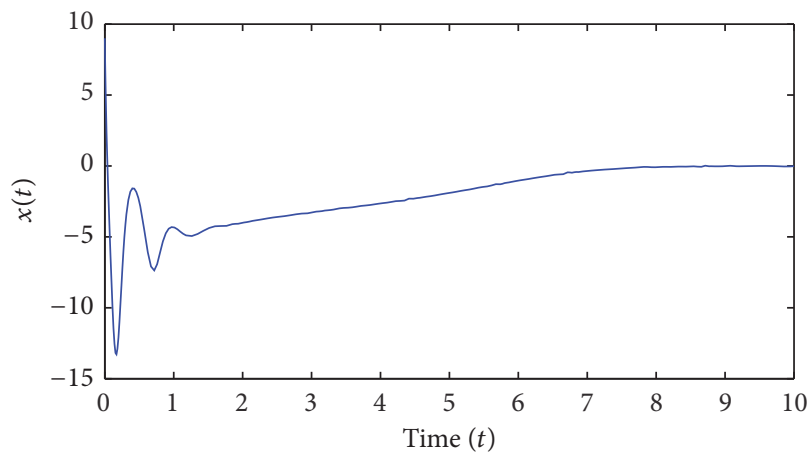

(a)

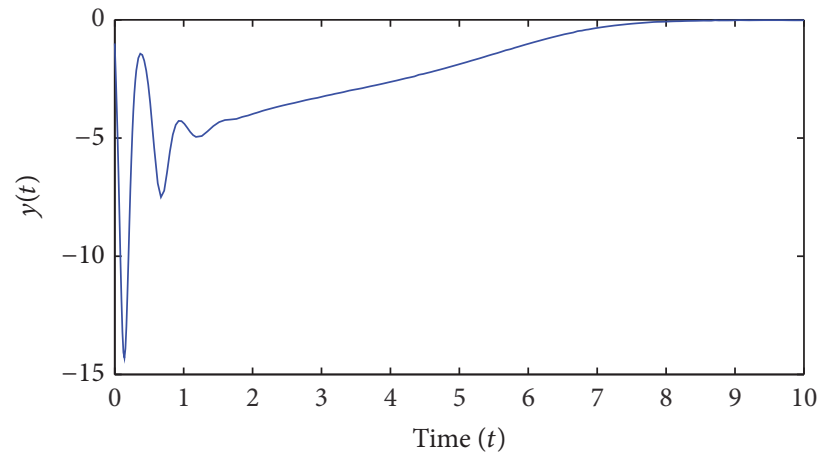

(b)

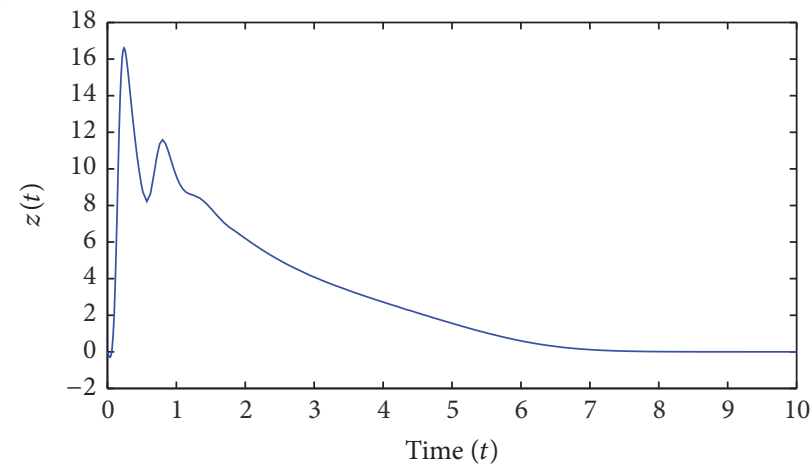

(c)

FIGURE 4: The PID control effect on Chen chaotic system from initial states $x_{1}(0)=9, x_{2}(0)=-1$, and $x_{3}(0)=0$ with an internal disturbing signal $\omega=\sin (t)$. In it, (a) the control effect of PID on $x_{1}$ variable of Chen chaotic system; (b) the control effect of PID on $x_{2}$ variable of Chen chaotic system; and (c) the control effect of PID on $x_{3}$ variable of Chen chaotic system. 


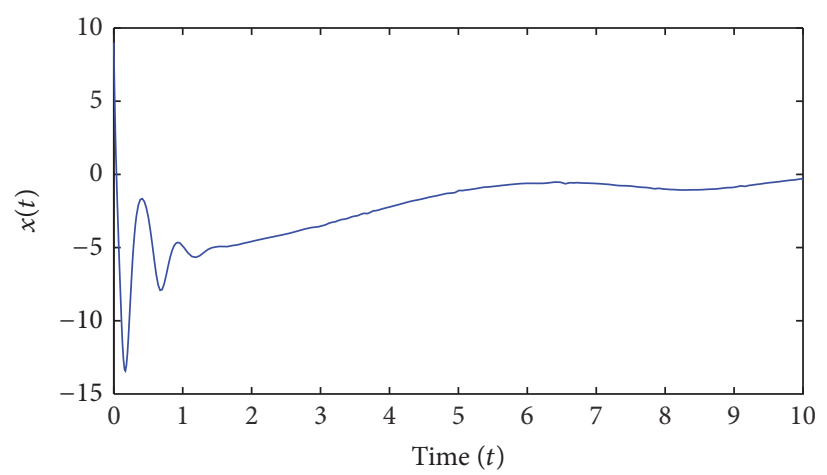

(a)

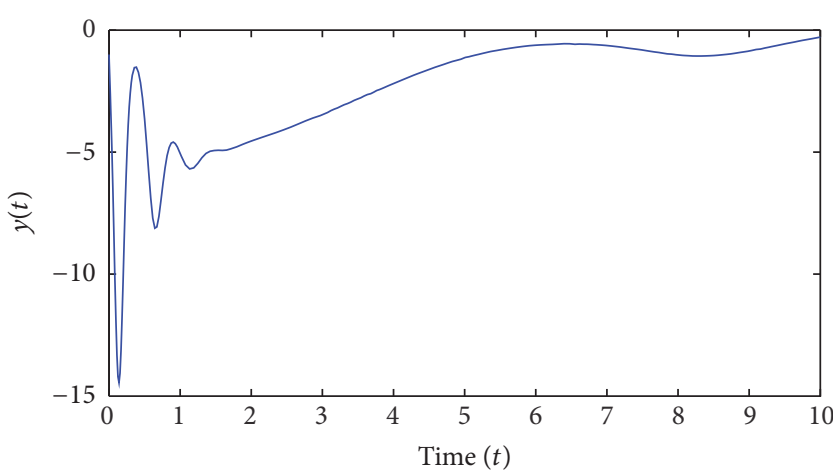

(b)

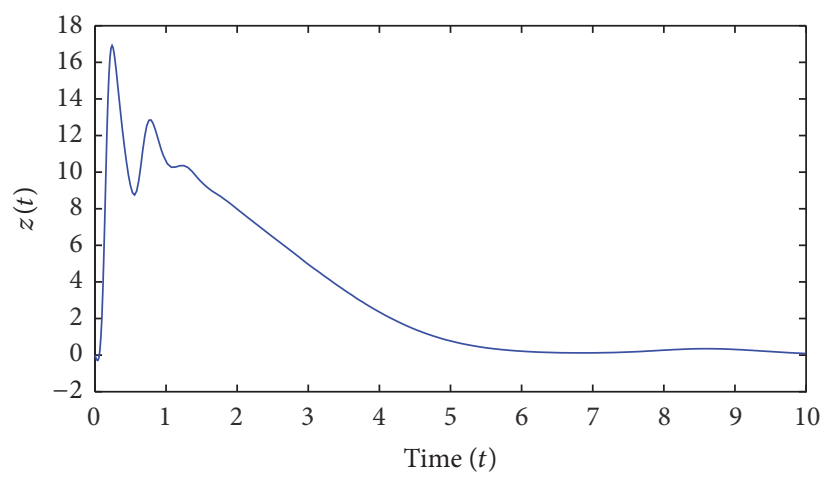

(c)

FIgURE 5: The PID control effect on Chen chaotic system from initial states $x_{1}(0)=9, x_{2}(0)=-1$, and $x_{3}(0)=0$ with an external disturbing signal $\omega=\sin (t)$. In it, (a) the control effect of PID on $x_{1}$ variable of Chen chaotic system; (b) the control effect of PID on $x_{2}$ variable of Chen chaotic system; (c) the control effect of PID on $x_{3}$ variable of Chen chaotic system.

control object system of a cascaded integral form is obtained as

$$
\begin{aligned}
\dot{y}_{1}= & y_{2}, \\
\dot{y}_{2}= & y_{3}, \\
\dot{y}_{3}= & 1925 x_{1}-14210 x_{2}+385 x_{1} x_{3}-1225 x_{2} x_{3} \\
& -35 x_{1}^{2} x_{2}-35 x_{1} u, \\
y= & y_{1} .
\end{aligned}
$$

Then, the variables of Chen chaotic system can be directly tracked by using the ADRC controller.

The adopted ADRC algorithm is shown in [24, 25, 27]. The controller parameters are set: $h_{0}=0.001, r_{0}=10000$, $\beta_{01}=1000, \beta_{02}=30000, \beta_{03}=800000, r=3000, c=3$, and $h=0.08$.

\subsection{The ADRC Control Effect for the Converted System}

(1) When the internal/external disturbances are all $\omega=0$, and the ADRC controller parameters are $h_{0}=0.001$, $r_{0}=10000, \beta_{01}=1000, \beta_{02}=30000, \beta_{03}=800000$, $r=3000, c=3, h=0.08$, the best control effect that can be achieved for Chen chaotic system from initial states $x_{1}(0)=9, x_{2}(0)=-1$, and $x_{3}(0)=0$ is shown in Figure 6.
(2) Under the same ADRC controller and its parameter settings, when there is an internal disturbance signal of $\omega=\sin (t)$, the best control effect that can be achieved for Chen chaotic system from initial states $x_{1}(0)=9, x_{2}(0)=-1$, and $x_{3}(0)=0$ is shown in Figure 7.

(3) Under the same ADRC controller and its parameter settings, when there is an external disturbance signal of $\omega=\sin (t)$, the best control effect that can be achieved for Chen chaotic system from initial states $x_{1}(0)=9, x_{2}(0)=-1$, and $x_{3}(0)=0$ is shown in Figure 8.

From the simulation results, it can be seen that converting control system, expressed as state space form or as state space of noncascaded integral form, into cascaded integral form controlled with ADRC is feasible not only in theory but also in practice. In addition, it is also very easy for the ADRC controller to achieve much better control results than the corresponding PID controller, especially in terms of control speed and overshoot performance, after the system conversion.

\section{Conclusions}

This paper first introduces the ADRC method and its typical form of cascaded integral system. The actual application 


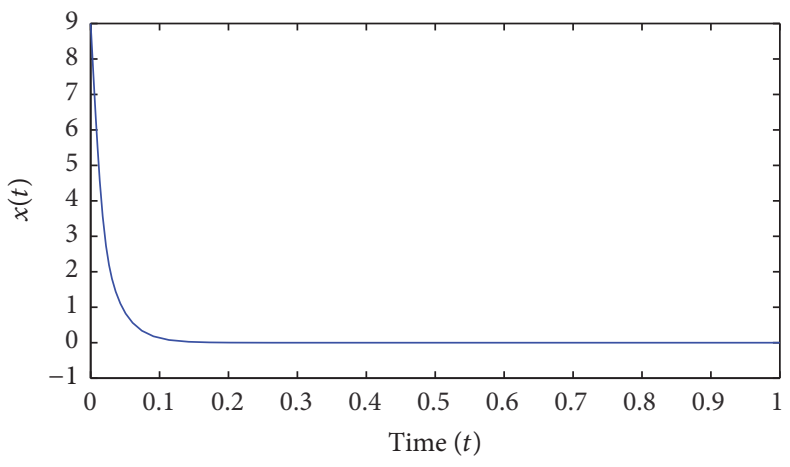

(a)

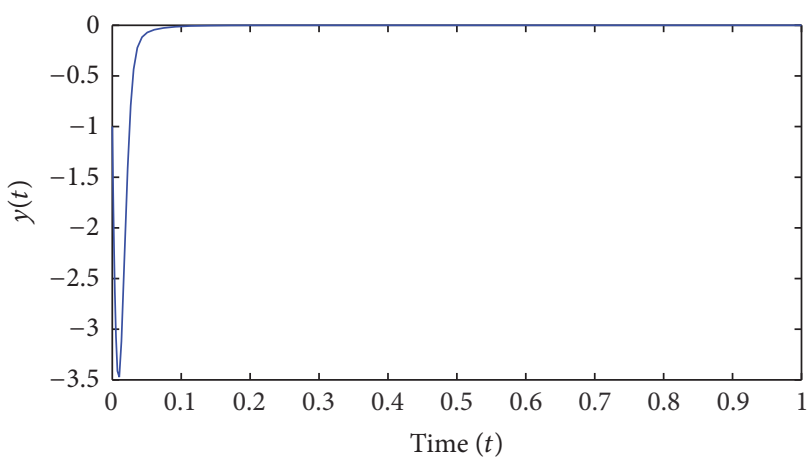

(b)

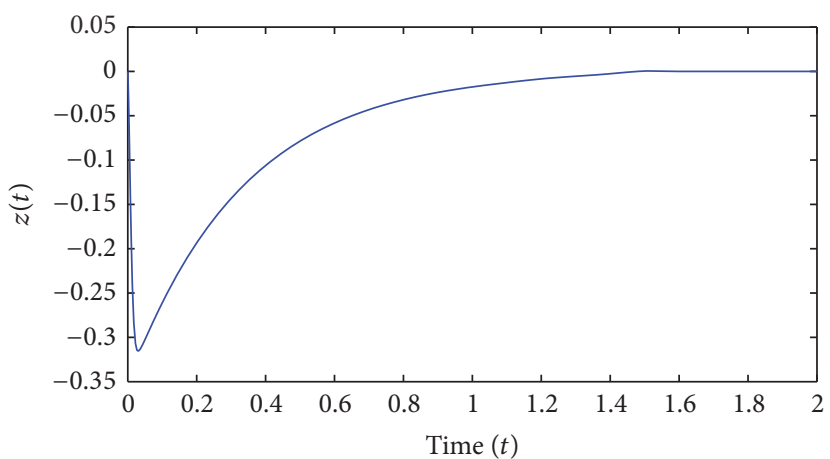

(c)

FIGURE 6: The ADRC control effect on Chen chaotic system from initial states $x_{1}(0)=9, x_{2}(0)=-1$, and $x_{3}(0)=0$ without disturbing. In it, (a) the control effect of ADRC on $x_{1}$ variable of Chen chaotic system; (b) the control effect of ADRC on $x_{2}$ variable of Chen chaotic system; and (c) the control effect of ADRC on $x_{3}$ variable of Chen chaotic system.

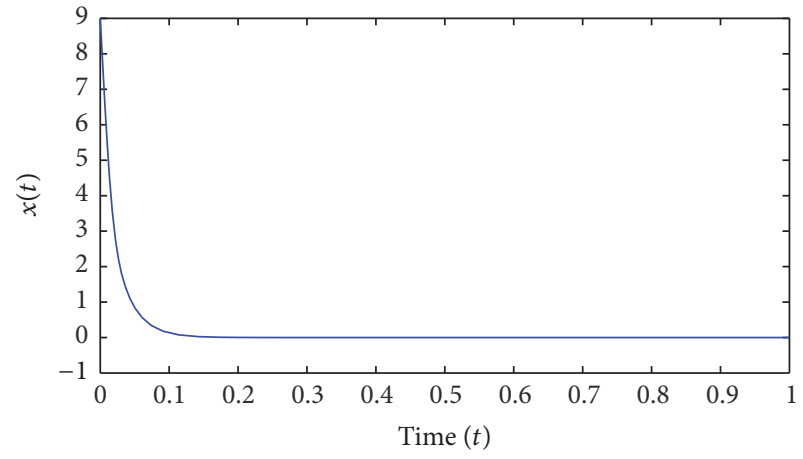

(a)

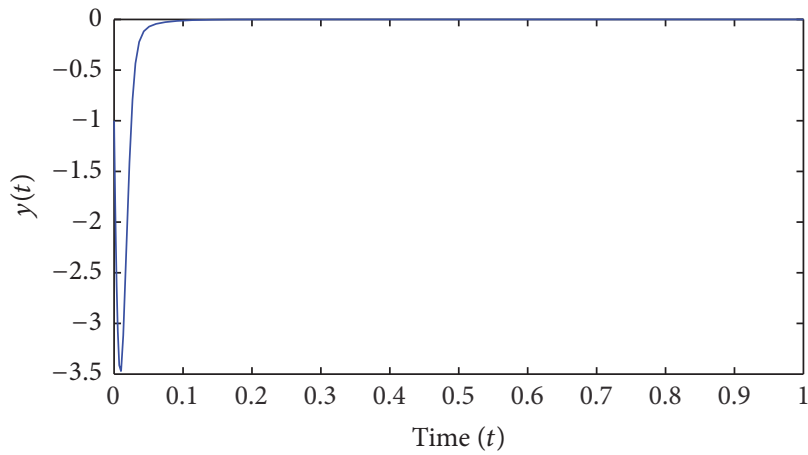

(b)

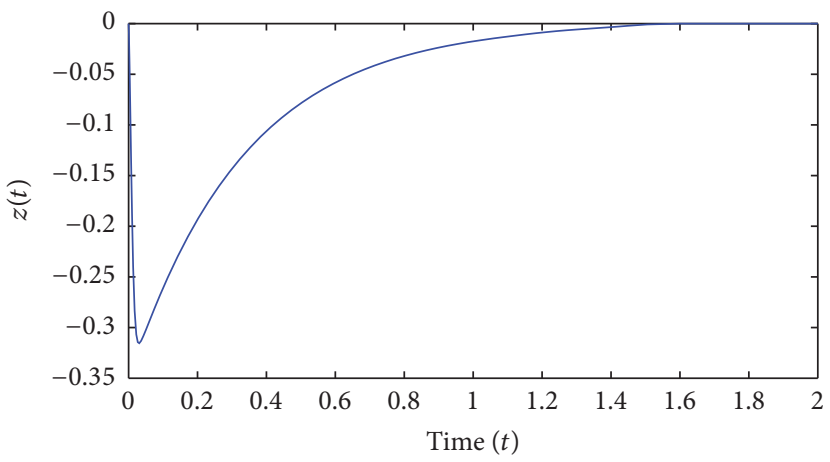

(c)

FIgURE 7: The ADRC control effect on Chen chaotic system from initial states $x_{1}(0)=9, x_{2}(0)=-1$, and $x_{3}(0)=0$ with an internal disturbing signal $\omega=\sin (t)$. In it, (a) the control effect of ADRC on $x_{1}$ variable of Chen chaotic system; (b) the control effect of ADRC on $x_{1}$ variable of Chen chaotic system; and (c) the control effect of ADRC on $x_{3}$ variable of Chen chaotic system. 


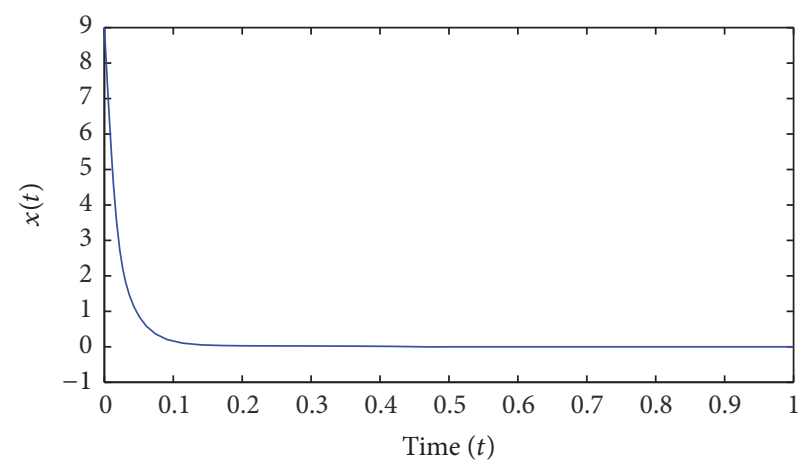

(a)

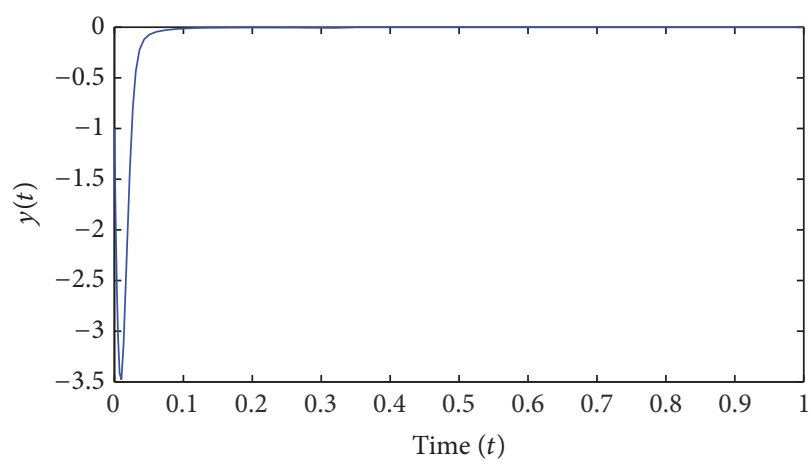

(b)

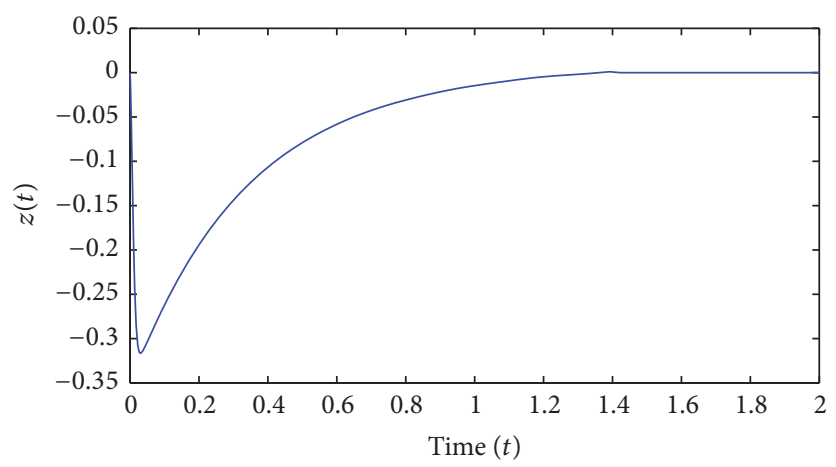

(c)

Figure 8: The ADRC control effect on Chen chaotic system from initial states $x_{1}(0)=9, x_{2}(0)=-1$, and $x_{3}(0)=0$ with an external disturbing signal $\omega=\sin (t)$. In it, (a) the control effect of ADRC on $x_{1}$ variable of Chen chaotic system; (b) the control effect of ADRC on $x_{2}$ variable of Chen chaotic system; and (c) the control effect of ADRC on $x_{3}$ variable of Chen chaotic system.

systems have various forms and do not necessarily have the characteristics of the cascaded integral system, especially the most typical state space control system. In this way, this paper presents a kind of method converting the noncascaded integral system into cascaded integral form. Then, the converted system can be controlled directly with ADRC method. Meanwhile, take the Chen chaotic system as an example and convert it into cascaded integral system adopting the method of the total derivative of composite functions of several variables and the structure. Then study comparatively the control effect between the original control system with PID and the converted control system with ADRC, under no disturbance as well as a variety of internal and external disturbance tests, while keeping the controller design and its parameters unchanged. It is very easy for the ADRC controller to achieve much better control results than the corresponding PID controller, especially in the aspect of control speed and overshoot performance. Thus, the research results are shown: (1) this converting method is not only correct in theory but also feasible in practice; (2) with converting control system, expressed as state space form or as state space form, into cascaded integral form controlled with ADRC, it is also very easy to achieve much better control results than the corresponding PID controller; (3) for state space object system of noncascaded integral with unknown object model, it can still realize the conversion and good ADRC control effect, as long as the system order can be determined; (4) the error of the object model can be seen as an internal or external disturbance for ADRC controller and then be estimated and compensated by the ESO, which is also a great advantage of ADRC controller; (5) this conversion method can solve the control problem of much noncascaded integral systems in theory and application and greatly expands the application scope of the standard ADRC method.

\section{Competing Interests}

The authors declare that they have no competing interests.

\section{Acknowledgments}

This work is supported by the NSFC projects of China under Grants nos. 61403250 and 51509151, the Bureau Project of China under Grant no. 2015HT056, and the Science Commission of Shanghai under Grant no. 13510501600.

\section{References}

[1] Q. Zheng, Z. Chen, and Z. Gao, "A practical approach to disturbance decoupling control," Control Engineering Practice, vol. 17, no. 9, pp. 1016-1025, 2009.

[2] K. Chang, Y. Xia, K. Huang, and D. Ma, "Obstacle avoidance and active disturbance rejection control for a quadrotor," Neurocomputing, vol. 190, pp. 60-69, 2016. 
[3] D. Wu, T. Zhao, K. Chen, and X. Wang, "Application of active disturbance rejection control to variable spindle speed noncircular turning process," International Journal of Machine Tools and Manufacture, vol. 49, no. 5, pp. 419-423, 2009.

[4] M. Ran, Q. Wang, and C. Dong, "Stabilization of a class of nonlinear systems with actuator saturation via active disturbance rejection control," Automatica, vol. 63, pp. 302-310, 2016.

[5] R. Shi, T. He, J. Peng, Y. Zhang, and W. Zhuge, "System design and control for waste heat recovery of automotive engines based on Organic Rankine Cycle," Energy, vol. 102, pp. 276-286, 2016.

[6] H. Zhang, J. Wang, G. Chen, and C. Yan, "A new hybrid control scheme for an integrated helicopter and engine system," Chinese Journal of Aeronautics, vol. 25, no. 4, pp. 533-545, 2012.

[7] X. Yang, J. Cui, D. Lao, D. Li, and J. Chen, "Input Shaping enhanced Active Disturbance Rejection Control for a twin rotor multi-input multi-output system (TRMS)," ISA Transactions, vol. 62, pp. 287-298, 2016.

[8] Y. Sun, W. Liu, M. Su, X. Li, H. Wang, and J. Yang, "A unified modeling and control of a multi-functional current sourcetyped converter for V2G application," Electric Power Systems Research, vol. 106, pp. 12-20, 2014.

[9] A. Noshadi and M. Mailah, "Active disturbance rejection control of a parallel manipulator with self learning algorithm for a pulsating trajectory tracking task," Scientia Iranica, vol. 19, no. 1, pp. 132-141, 2012.

[10] D. Wu, K. Chen, and X. Wang, "Tracking control and active disturbance rejection with application to noncircular machining," International Journal of Machine Tools \& Manufacture, vol. 47, no. 15, pp. 2207-2217, 2007.

[11] T. Jiang, C. Huang, and L. Guo, "Control of uncertain nonlinear systems based on observers and estimators," Automatica. A Journal of IFAC, the International Federation of Automatic Control, vol. 59, pp. 35-47, 2015.

[12] W. Tan, Y. Hao, and D. Li, "Load frequency control in deregulated environments via active disturbance rejection," International Journal of Electrical Power and Energy Systems, vol. 66, pp. 166-177, 2015.

[13] Z. Lei and C. Guo, "Disturbance rejection control solution for ship steering system with uncertain time delay," Ocean Engineering, vol. 95, pp. 78-83, 2015.

[14] J. Li, X. Qi, Y. Xia, F. Pu, and K. Chang, "Frequency domain stability analysis of nonlinear active disturbance rejection control system," ISA Transactions, vol. 56, pp. 188-195, 2015.

[15] J. Song, L. Wang, G. Cai, and X. Qi, "Nonlinear fractional order proportion-integral-derivative active disturbance rejection control method design for hypersonic vehicle attitude control," Acta Astronautica, vol. 111, article no. 5364, pp. 160169, 2015.

[16] C.-E. Huang, D. Li, and Y. Xue, "Active disturbance rejection control for the ALSTOM gasifier benchmark problem," Control Engineering Practice, vol. 21, no. 4, pp. 556-564, 2013.

[17] D. Li, C. Li, Z. Gao, and Q. Jin, "On active disturbance rejection in temperature regulation of the proton exchange membrane fuel cells," Journal of Power Sources, vol. 283, pp. 452-463, 2015.

[18] Z.-L. Zhao and B.-Z. Guo, "On active disturbance rejection control for nonlinear systems using time-varying gain," European Journal of Control, vol. 23, pp. 62-70, 2015.

[19] S. Balajiwale, H. Arya, and A. Joshi, "Study of performance of ADRC for longitudinal control of MAV,' IFAC-PapersOnLine, vol. 49, no. 1, pp. 585-590, 2016.
[20] G. Shen, Y. Xia, and H. Sun, "A 6DOF mathematical model of parachute in Mars EDL," Advances in Space Research, vol. 55, no. 7, pp. 1823-1831, 2015.

[21] Q. Chen, L. Li, M. Wang, and L. Pei, "The precise modeling and active disturbance rejection control of voice coil motor in high precision motion control system," Applied Mathematical Modelling, vol. 39, no. 19, pp. 5936-5948, 2015.

[22] Y. Shen, K. Shao, W. Ren, and Y. Liu, "Diving control of autonomous underwater vehicle based on improved active disturbance rejection control approach," Neurocomputing, vol. 173, pp. 1377-1385, 2016.

[23] Y. Wu and Q. Zheng, "ADRC or adaptive controller-a simulation study on artificial blood pump," Computers in Biology and Medicine, vol. 66, pp. 135-143, 2015.

[24] J. Han, "Active disturbance rejection controller and its applications," Control Decision, vol. 13, no. 1, pp. 19-23, 1998 (Chinese).

[25] J. Han, "From PID to active disturbance rejection control," IEEE Transactions on Industrial Electronics, vol. 56, no. 3, pp. 900 906, 2009.

[26] A. Isidori, Nonlinear Control System, Springer, London, UK, 3rd edition, 1995.

[27] Y. Huang and W. Xue, "Active disturbance rejection control: methodology and theoretical analysis," ISA Transactions, vol. 53, no. 4, pp. 963-976, 2014.

[28] M. Ramírez-Neria, H. Sira-Ramírez, R. Garrido-Moctezuma, and A. Luviano-Juárez, "Linear active disturbance rejection control of underactuated systems: the case of the Furuta pendulum," ISA Transactions, vol. 53, no. 4, pp. 920-928, 2014.

[29] Z. Gao, "Scaling and bandwidth-parameterization based controller tuning," in Proceedings of the American Control Conference, pp. 4989-4996, IEEE, Denver, Colo, USA, 2003.

[30] Z. Gao, "Active disturbance rejection control: a paradigm shift in feedback control system design," in Proceedings of the American Control Conference, pp. 2399-2405, Minneapolis, Minn, USA, 2006.

[31] D. Wu and K. Chen, "Frequency-domain analysis of nonlinear active disturbance rejection control via the describing function method," IEEE Transactions on Industrial Electronics, vol. 60, no. 9, pp. 3906-3914, 2013.

[32] H. Feng and B.-Z. Guo, "Output feedback stabilization of an unstable wave equation with general corrupted boundary observation," Automatica, vol. 50, no. 12, pp. 3164-3172, 2014.

[33] L. Sun, D. Li, Z. Gao, Z. Yang, and S. Zhao, "Combined feedforward and model-assisted active disturbance rejection control for non-minimum phase system," ISA Transactions, vol. 64, pp. 24-33, 2016.

[34] Department of Applied Mathematics of Tongji University, Higher Mathematics (2), Version 5, Higher Education Press, Beijing, China, 2008.

[35] Z. Yan, "Controlling hyperchaos in the new hyperchaotic Chen system," Applied Mathematics and Computation, vol. 168, no. 2, pp. 1239-1250, 2005. 


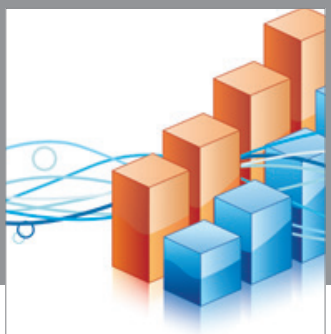

Advances in

Operations Research

vatem alat4

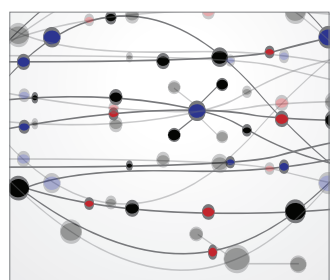

\section{The Scientific} World Journal
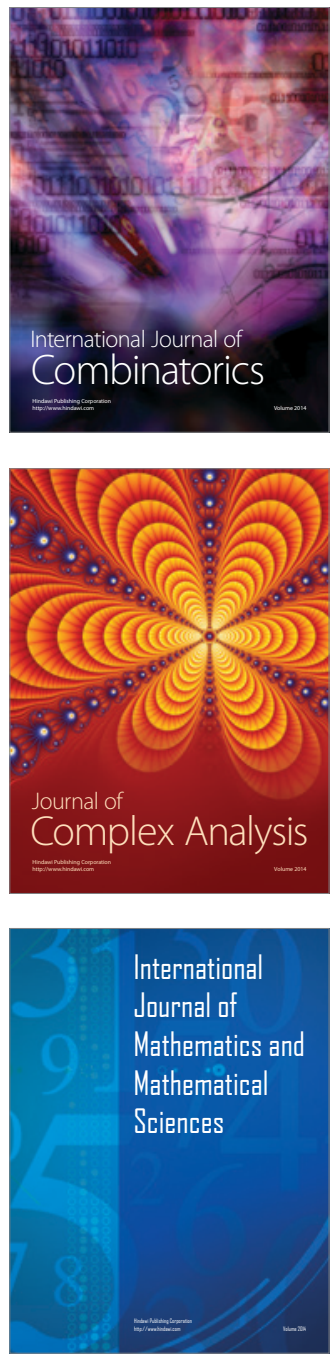
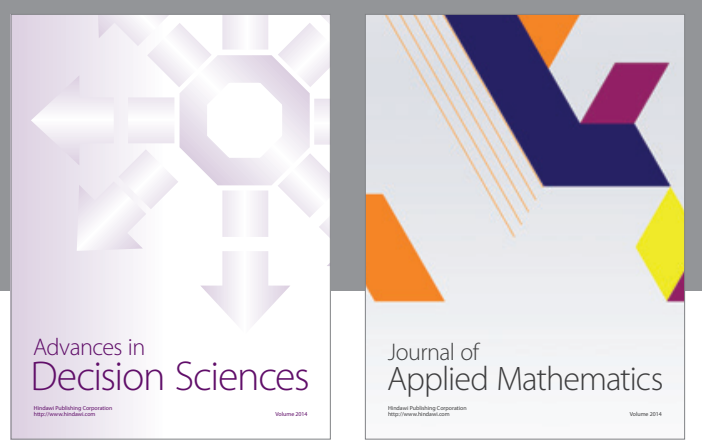

Algebra

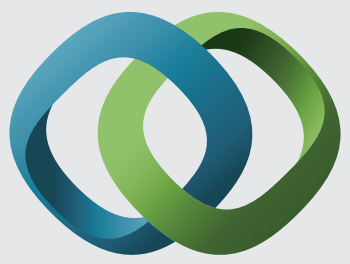

\section{Hindawi}

Submit your manuscripts at

http://www.hindawi.com
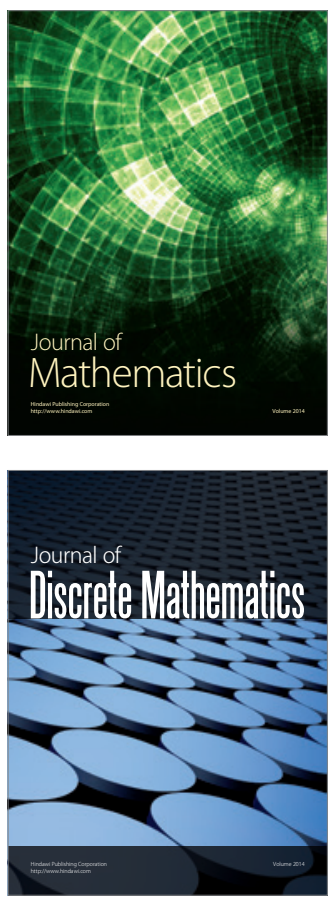

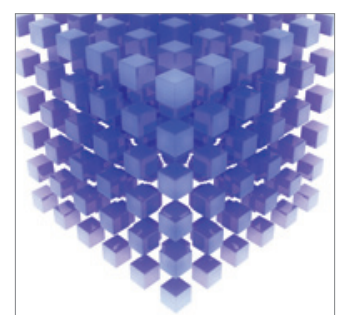

Mathematical Problems in Engineering
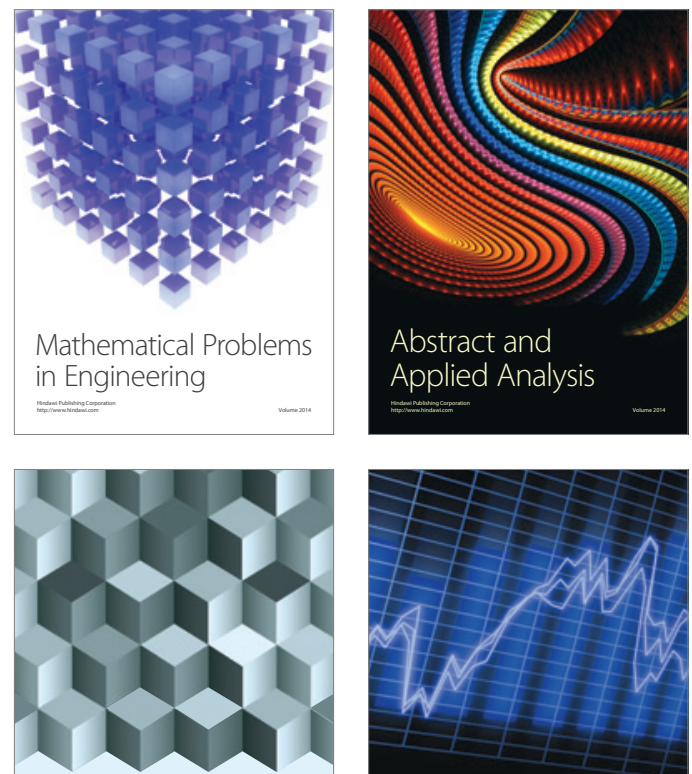

Journal of

Function Spaces

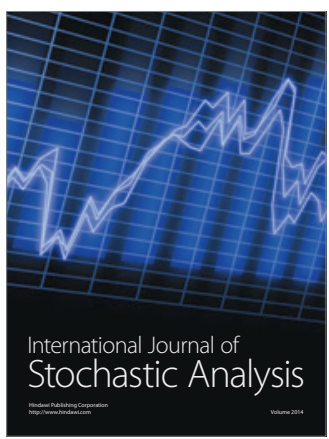

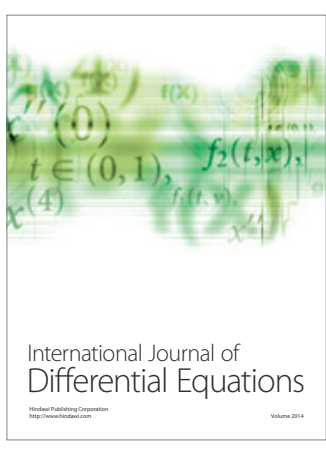
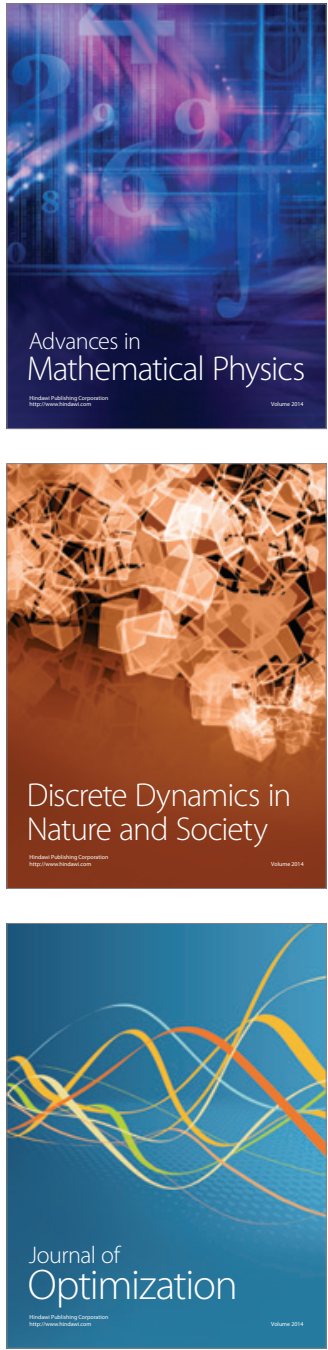